\title{
Unveiling the elasticity of individual aortic fiber during ageing at nanoscale by in situ Atomic Force Microscopy
}

Received 00th January 20xx, Accepted 00th January 20xx

DOI: $10.1039 / \times 0 \times x 00000 x$

\author{
Alexandre Berquand ${ }^{1,2, \neq}$, Amandine Wahart ${ }^{3, \neq}$, Aubéri Henry $^{3}$, Laetitia Gorisse ${ }^{3}$, Pascal Maurice ${ }^{3}$, \\ Sébastien Blaise ${ }^{3}$, Béatrice Romier-Crouzet ${ }^{3}$, Christine Pietrement ${ }^{3,4}$, Amar Bennasroune $^{3}$, Hervé \\ Sartelet ${ }^{3}$, Stéphane Jaisson ${ }^{3,5}$, Philippe Gillery ${ }^{3,5}$, Laurent Martiny ${ }^{3}$, Fatouma Touré ${ }^{6,7}$, Laurent \\ Duca $^{3, \#^{*}}$, Michael Molinari ${ }^{8, \#^{*}}$
}

\section{Introduction}

The elastic fiber fate is of prime importance in many cardiovascular diseases and their loss of functionalities is linked the alteration of their mechanical properties with ageing ${ }^{1}$. For example, it has been clearly shown that elastin deficiency leads to arterial stiffening and hypertension, and that the latter can be highly involved in atherosclerosis development because of

1 Laboratoire de Recherche en Nanosciences, LRN EA4682, University of Reims Champagne-Ardenne, 51685 Reims, France.

2 Nano'Mat platoform, University of Reims Champagne-Ardenne, 51100 Reims, France.

3 MEDYC UMR CNRS 7369, "Matrice Extracellulaire et Dynamique Cellulaire", University of Reims Champagne-Ardenne, 51100 Reims, France, Team 2 "Matrix Ageing and Vascular Remodelling"

4 University Hospital of Reims, Department of Pediatrics (Nephrology unit), Reims, France.

5University Hospital of Reims, Department of Biochemistry-PharmacologyToxicology, Reims, France.

6 University Hospital of Limoges, (Nephrology unit), Limoges, France.

7 CRIBL UMR CNRS 7276-INSERM U1262 "Contrôle de la Réponse Immune et Lymphoproliférations", University of Limoges, 87025 Limoges, France

8 CBMN, CNRS UMR 5248, IPB, Université de Bordeaux, 33607 Pessac, France.

* Corresponding authors: michael.molinari@u-bordeaux.fr, laurent.duca@univreims.fr

$\#, \ddagger$ These authors contributed equally to the work the subsequent damages made to arteries making them more prone to atherosclerotic plaques build up ${ }^{2,3}$. Indeed, the resilience of vertebrate tissues is due to the presence of elastic fibers in their extracellular environment ${ }^{4}$. They are made of macromolecular assemblies of tropoelastin and glycoproteins synthesized by various cells such as fibroblasts or smooth muscle cells ${ }^{5}$, which provide elasticity to tissues such as arteries as a response to a physical stress ${ }^{6}$. The sub-cellular network of elastic fibers is tissue-dependent and in the vessel wall, they form concentric cylinders called lamellae, which surround the vessels' lumen. This lamellae architecture is essential to ensure the flexibility, compliance and integrity of the vessel in response to a physical stress like the blood flow ${ }^{5}$. Due to its specific architecture made of cross-linked polymers of tropoelastin molecules covalently bound to each-other with a high reticulation rate, elastin is a remarkably stable molecule ${ }^{7}$. This protein has a very low renewal rate, and is subject to degradation by enzymes called elastases and to chemical modifications such as glycation increasing together with the ageing process ${ }^{8,9}$. It has been described that during ageing, the degradation of elastic fibers along with the increase and remodeling of vascular collagen highly participate to the appearance of vascular stiffness. This results in the loss of the capacity of the vessel to respond to a physical stress, associated to systolic hypertension, higher heart fatigue, coronary artery 
disease, stroke or atrial fibrillation ${ }^{10,11}$. However, the molecular cause of arterial stiffness is far more complex and elastin could lose its elasticity ability without being degraded due, for instance, to the above mentioned chemical modifications. Consequently, comparative studies of the elastic fiber and its surrounding matrix mechanics between young fibers and aged ones represent a very promising approach to better understand the evolution of the arterial stiffness and to explore novel diagnosis methods. A wide variety of experimental tools has been developed to study the fiber morphologies and mechanics at different length and time scales but the spatial resolution remains limited for most of them, including optical and fluorescence measurements and the correlation with the mechanical properties is not direct and simultaneous as the methods used for such measurements are not performed at the same time. For instance, aortic Pulse Wave Velocity (aPWV) is used as an indirect measurement of the vessel stiffness but with an average response of a macroscopic system which limits its utility when investigating individual fiber. Fluorescence microscopy is used to visualize elastin degradation but with limited lateral resolution, which does not allow the differentiation of the fiber components and does not give any information about the fiber elasticity. Scanning Acoustic Microscopy, based on the speed estimation of longitudinal acoustic waves sent through a material, could be used to study the elastic properties of aorta but with a micrometer lateral resolution limiting its interest ${ }^{12-14}$. Atomic Force Microscopy (AFM) has lots of advantages as it allows to obtain high resolution imaging in physiological medium and simultaneously, to investigate the mechanical properties of biological objects at the nanoscale. The ability of AFM as a spectroscopic tool to obtain the mechanical response of elastic fibers has led to different studies but still with limited spatial resolution around the $\mu \mathrm{m}$ when it comes to characterize their elastic properties. Among the studies involving AFM measurements, Tracqui et al. 15 have taken advantage of the possibility to get quantitative mechanical information using Force-Distance (FD) curves coming from the interaction of a polymeric bead of a few microns in diameter with specific places of atherosclerotic plaques localized with optical microscopy after staining. Using proper mechanical models, the FD curves have been fitted to describe the elastic response of the probed objects and to get their apparent or relative Young's modulus. In other papers, Frequency-modulated atomic force microscopy (FM-AFM), which relies on the detection of small changes in the cantilever resonance frequency happening when the tip-sample interactions are modified in response to a local change in mechanical or viscoelastic properties ${ }^{16}$ is also commonly used to investigate this type of samples ${ }^{17}$. For instance, it has been used as a spectroscopic tool to assess the viscoelastic remodeling of sheep aorta of various age ${ }^{18}$. Those AFM-related measurements techniques, have only been used to perform mechanical spectroscopy but a cartography of the fiber's elasticity, which is of prime importance to precisely characterize the fiber's behavior, was not achieved. This important drawback is strongly limiting for correlation studies at it is difficult to know exactly where the different measurements have been carried out. It is then important to improve these kinds of measurements to get reliable mapping at high spatial resolution and to get quantitative signals, in order to better understand the structure-function relationships within arteries. Recent instrumental developments in AFM have led to improved resolution, better accuracy and consistency in the mechanical measurements in correlation with the classical topographical images ${ }^{19}$. Based on the merging of the classical Force-Volume mode, which was limited by its resolution and its acquisition speed, and the dynamical modes, quantitative nanomechanical modes such as the PeakForce Quantitative NanoMechanical imaging of Bruker (PFQNM) have been developed and applied to biological objects such as cells. This oscillating technique is based on an off-resonance of the cantilever, allowing simultaneous acquisition of topographical and mechanical information in a semi-quantitative or quantitative manner (when the probe is calibrated prior to the experiments) and at resolutions comparable to those of tapping mode AFM. If such modes have been successfully used to image soft biological samples like live cells ${ }^{20,21}$, no tissue characterizations have been performed since sample preparation, image acquisition and quantitative measurements on tissues as aorta are not trivial issues.

In this study, the PFQNM mode was used to study at high resolution, the morphologies and the nanomechanical properties of aorta from mice of different ages and the results were compared with data coming from classical techniques such as fluorescence and aPWV. Thanks to a proper sample preparation and careful AFM measurements, simultaneous images of the topography of aorta sections and of their mechanical properties have been obtained at the nanoscale, especially those of elastic fibers, allowing to highlight at high resolution the relationships between the structure and the function of such fibers together with the ageing process and the related arterial stiffness.

\section{Results and discussion}

During ageing, vascular stiffness appears to be due to a complex sequence of events. One of the main involved processes is the extracellular matrix remodeling, showing a clear increase in collagen content and in elastin degradation. However, the molecular elasticity of the remaining elastic fibers has never been considered and could be a crucial determinant.

Table 1: Evolution of the aPWV values with mice ageing $(p<0.001$ for each intervals).

\begin{tabular}{|c|c|c|c|c|}
\hline $\begin{array}{c}\text { Mice Age } \\
\text { (months) }\end{array}$ & 1 & 5 & 10 & 20 \\
\hline $\begin{array}{c}\text { aPWV } \\
\left(\mathrm{cm} . \mathrm{s}^{-1}\right)\end{array}$ & $0.198 \pm 0.015$ & $0.223 \pm 0.019$ & $0,248 \pm 0.027$ & $0.343 \pm 0.024$ \\
\hline
\end{tabular}


The development of an in situ nanoscale characterization of individual elastic fiber molecular stiffness brings crucial information about that, as well as high resolution images of elastic fibers topology. We used mice aged from 1 month (V1) to 20 months (V20) and first performed aPWV to show the appearance of the expected vascular stiffness. Indeed, aPWV is commonly used for measuring the speed of propagation of the aortic pulse wave, which is a reflection of arterial stiffness. As expected, our results showed a clear and significant increase of the aPWV with ageing from V1 to V20 (table 1).
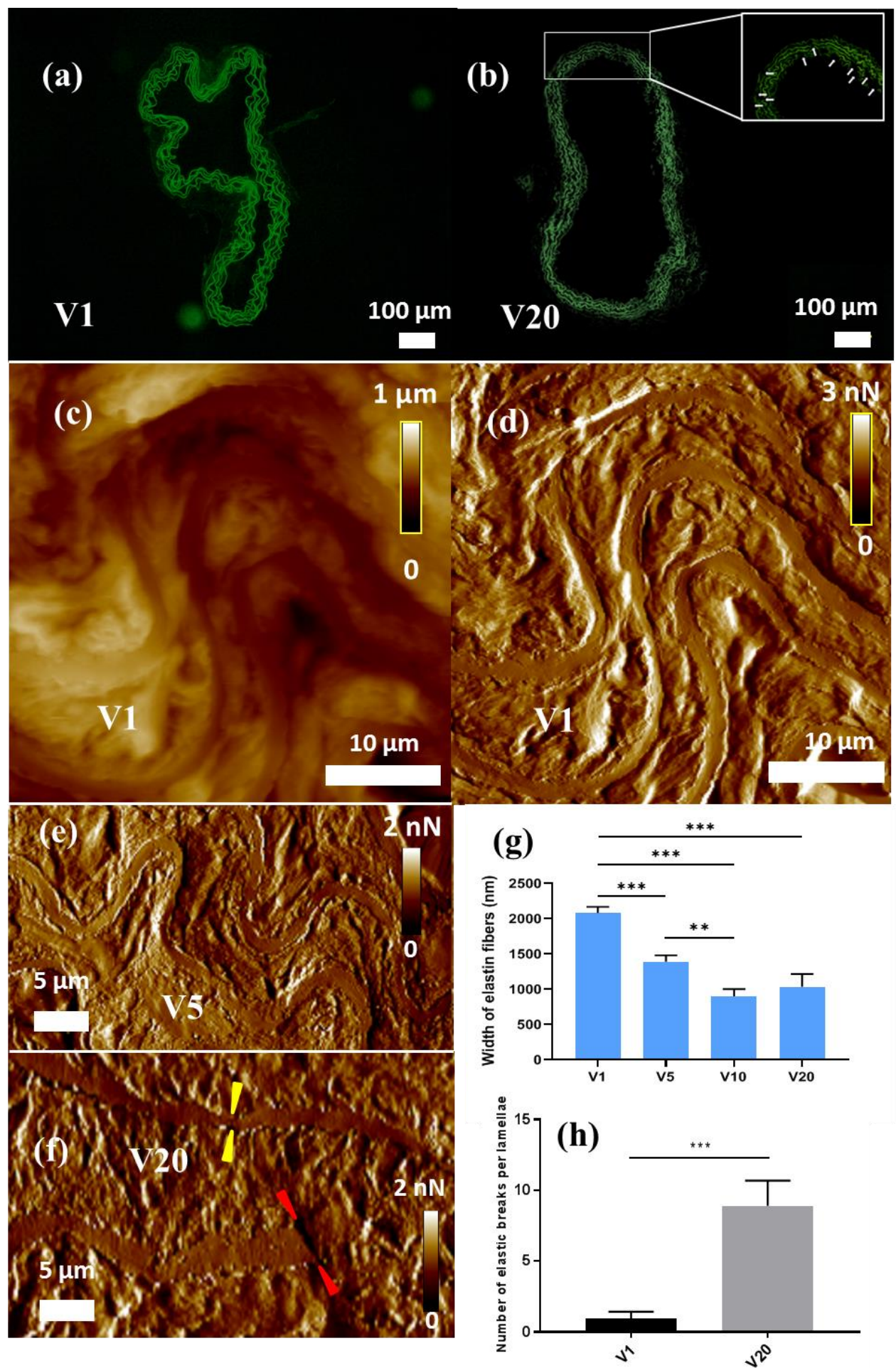

Figure 1. Evaluation of elastic fibers breaks and width using fluorescence microscopy and AFM analysis during ageing. (a) Fluorescence imaging of Aorta sections from V1 specimen, (b) for V20 specimen (c) AFM topographical image of Aorta sections from V1 specimen, (d) and associated AFM PeakForce Error image of Aorta sections from V1 specimen, (e) AFM PeakForce Error image of Aorta sections from V5 specimen, (f) AFM PeakForce Error image of Aorta sections from V20 specimen, (g) Histogram of the width of the elastic fibers taken from PeakForce images for the different conditions, ( $h$ ) Quantification of the elastic breaks per lamellae. On V1 can be seen thick and homogeneous elastin fibers parallel to each-other and very little extracellular matrix in between. On the contrary, the V20 image exhibit few fibers with highly heterogeneous thickness and rupture points. Results are expressed as mean $\pm \operatorname{SEM}(\mathrm{n}=15)^{* * *}, \mathrm{p}<0.001 ; * *, \mathrm{p}<0.01$ 
In order to correlate these data to the classically described elastin fragmentation observed during ageing, we analyzed elastin breaks using the autofluorescence of elastin. On Fig. 1a, the network of the elastic fibers on the aorta section from the V1 specimen clearly appears and presents no alteration. All the elastic lamellae are clearly visible without evident degradation. On Fig. 1b, the aortic elastic lamellae presents numerous breaks, classical features of vascular ageing linked to the appearance of vascular elasticity, previously demonstrated using aPWV. However, these classical observations only permit to describe macroscopic changes such as breaks and are too limited to precisely describe others parameters such as elastic fibers thickening or narrowing, for instance. They neither permit to clearly analyze nanoscale modifications both for the elastic lamellae and the elastic inter-fibers spaces, mainly composed of elastin, but also collagen and smooth muscle cells, that could also crucially impact vascular stiffness. Moreover, these classical descriptors do not allow to get valuable information about nanomechanical parameters. In this way, the AFM methods we developed are of high interest. Fig. 1c displays typical topography image from aorta sections from V1 specimen. On this image, elastic fibers and the elastic inter-fiber spaces could be evidenced with higher roughness for the inter-fiber spaces part. Even if the elastin fiber width could be evidenced on these images, the rugosity of the inter-fiber spaces is hiding some details on the topographical images. Then, PeakForce Error images were used (Fig. 1d). Since in PeakForce QNM the feedback is calculated from the maximal (so called Peak-) force applied to the sample, these data can be directly related to the local changes in topography but using Peakforce error images makes the elastic fiber features more easily distinguishable leading to easier quantification. On young specimens (V1), the elastic fibers exhibit parallel trajectories and are rather thick and homogeneous. The average space between the fibers is quite limited, which could explain that the overall tissue is more capable of damping the blood flow pressure and returning to its relaxation state. When increasing the mice age (Fig. 1e and f), on the peakforce error images, a decrease of the fiber width could be seen, coupled to a loss of homogeneity. This can be confirmed by the corresponding V20 image showing not only a high discrepancy in the fibers diameters (the yellow arrows show a typical shrinking point), but also rupture points (as shown in red). Such shrinking and rupture points were routinely observed on V20 samples whereas very hardly seen on V1 samples. The AFM resolution allows a quantification of these breaking points (Fig. 1h). This confirms the observation performed in fluorescence microscopy (Fig. 1b) where such rupture points are also evidenced for the V20 specimen due to elastin degradation but where the quantification is difficult regarding its lack of resolution. The interest of the AFM compared to the fluorescence images is that one can also visualized the inter-fiber space rugosities and features directly without labeling and that the fiber widths could be precisely quantified because of the AFM resolution. Interestingly, as can be seen on the histogram representation (Fig. 1g), the average diameter of elastic fibers decreases over time: $2.09 \pm 0.08 \mu \mathrm{m}$ for $\mathrm{V} 1,1.4 \pm 0.09$ $\mu \mathrm{m}$ for $\mathrm{V} 5,0.9 \pm 0.1 \mu \mathrm{m}$ for $\mathrm{V} 10$, and $1 \pm 0.2 \mu \mathrm{m}$ for $\mathrm{V} 20$. This decrease can be attributed to a progressive degradation of the fibers throughout the life not necessary leading to a break. Moreover, it is worthy to note that after 10 months, the mean width did not significantly evolve, although the SEM showed a higher dispersion of the measurements for V20, presumably due to a less homogenous level of degradation observed in advanced age. As a consequence, in older specimens, the elastic fibers are separated from each other by a much larger space than in young mice. Although macroscopic changes affecting elastic fibers during ageing have been described due to the modifications of the vascular walls (extra-cellular matrix remodeling, deposits of calcium or lipids, alterations due to repeated mechanical loading and oxidation stress leading to fragmentation and rupture ${ }^{22}$ ), fluorescence or histological analysis are inherently limited for a precise analysis. Our data lead to a deepened analysis thanks to the AFM resolution which allowed a precise characterization of the elastic fibers width. AFM demonstrated no significant changes happened after $\mathrm{V} 10$, suggesting that the majority of the modifications that elastic fibers suffered during ageing with an effect of their width should occur in the first half of life. Moreover, AFM studies allow a precise characterization of the nanomechanics of elastic fibers. As stated in the introduction, a huge potentiality of the AFM PFQNM mode is to simultaneously map different other properties such as the relative Young's modulus (YM) or the adhesion. Although various signals can be simultaneously extracted, the main interest of the study is to map in situ the elastic moduli of aorta sections. The evolution of the reduced Young's modulus along the ageing process for the V1, V5, V10, and V20 specimens was investigated. Fig. 2a exhibits a representative example of YM images that could be obtained using the PFQNM mode. On this image, for each point, a force curve is obtained and then, the value of the YM is extracted by fitting this force curve using the mechanical proper model. In our case, as seen on the Fig. $2 \mathrm{~b}$ with two representative curves extracted on elastic fibers (red curve) and the elastic interfiber space (blue curves), the adhesion component is negligible and then the Hertz model is perfectly fitting the curves. The YM image obtained for the V1 condition clearly exhibits a difference in the mechanical properties of the elastic fibers and on the elastic interfibers space. The average YM of elastic fibers was found to be $0.09 \pm$ $0.02 \mathrm{MPa}$ for V1 (Fig. 2f) with very homogeneous values along the fibers while the elastic inter-fiber space is softer with an elasticity of $40.8 \pm 9.26 \mathrm{kPa}$ (Fig. $2 \mathrm{~g}$ ). It is important to note that two distinct components, having very distinct mechanical properties, can be observed in between the fibers (fig. 2a). The darker components of the extracellular matrix have an average relative Young's modulus in the order of $10 \mathrm{kPa}$, which in agreement with values previously obtained on cultured rat aortic smooth muscle cells (SMCs) in culture (1.5 to $11 \mathrm{kPa})^{23}$, tends to prove that these very soft components could come from SMCs. Compared to other AFM studies ${ }^{11,14}$, using a mechanical mapping instead of performing local measurements using beads with a diameter superior to $5 \mu \mathrm{m}$ (ie superior to the elastin fiber width) allow to have more precise information and to discriminate the mechanical components of the elastic inter-fibers spaces and of the fibers. On Fig 2c-e, the evolution of the YM of the V5, V10, and V20 specimens has then been investigated. For the elastic fibers, an increase of the YM is observed with YM of $0.51 \pm$ 
$0.10 \mathrm{MPa}$ for $\mathrm{V} 5,1.20 \pm 0.33 \mathrm{MPa}$ for $\mathrm{V} 10$ and $2.50 \pm 0.26 \mathrm{MPa}$ for V20. The effect is quite pronounced between $\mathrm{V} 1$ and $\mathrm{V} 20$ conditions with an increase by a factor 28. This increase over time is in agreement with previous results obtained on different mammal
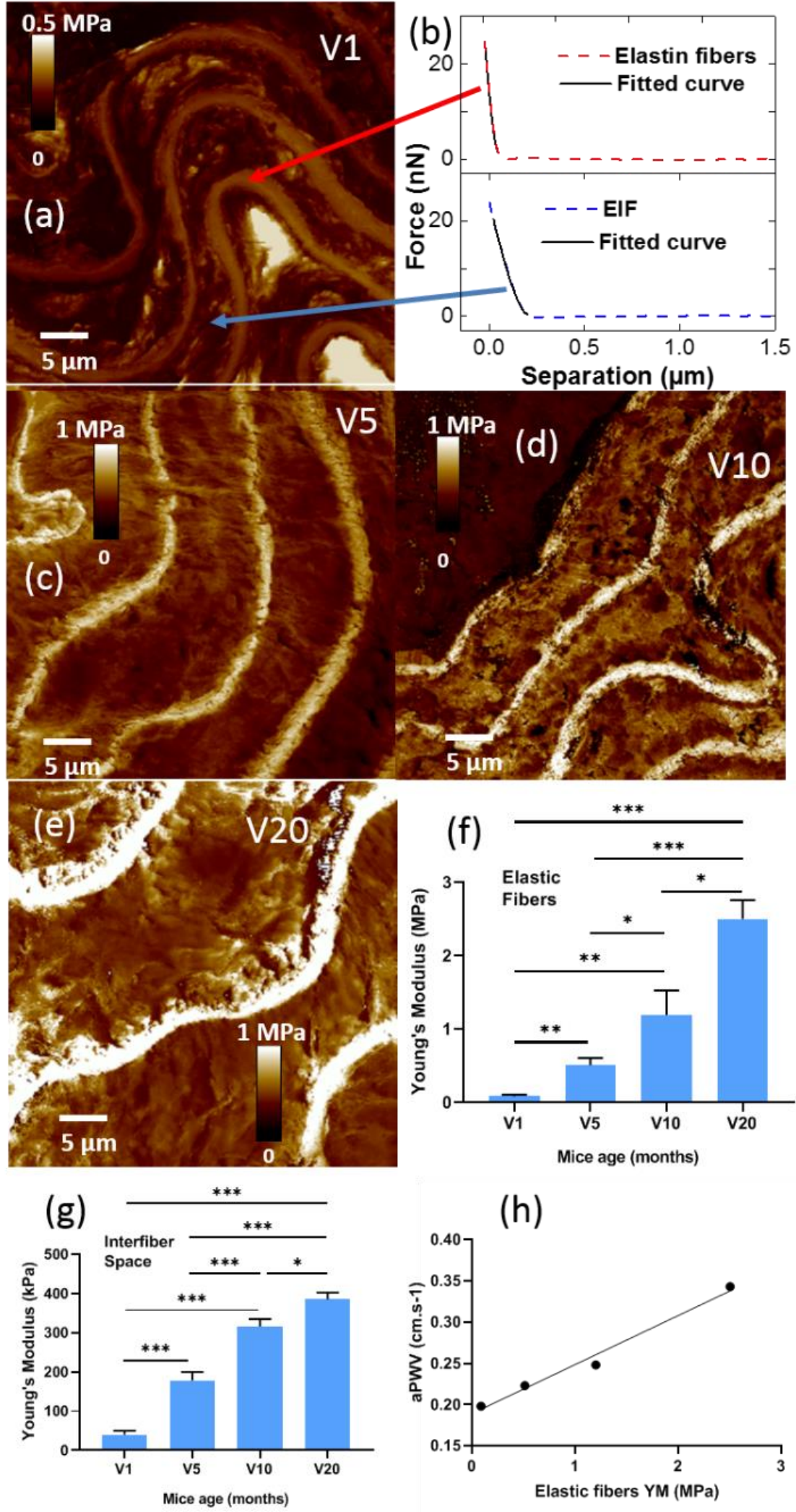

(h)

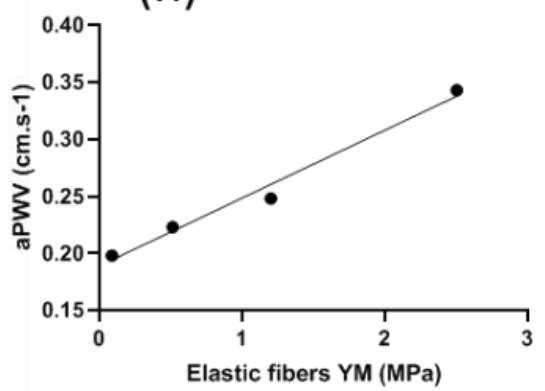

Figure 2. Determination of Young Modulus of elastic tibers and elastic inter-tiber space during ageing. (a) PFQNM YM images of Aorta section coming from the V1 specimen. (b) Typical force curves and their fits extracted from the image of the V1 specimen. The blue curve comes from a spot taken on the fiber while the red one is taken from the elastic inter-fiber space (EIF). On inset of the figure, representative retract and approach curves showing the elastic behaviour of the mechanical response; (c), (d) and (e) PFQNM YM image of Aorta coming from the V5, V10 and V20 samples, respectively. (f) Evolution of the relative Young's moduli of both elastic fibers, (g) elastic inter-fiber spaces over the ageing process, (h) linear correlation between aPWV and fibers relative Young's Modulus (Pearson correlation coefficient $r=0,9912) \quad(n=15) . * * *, p<0.001 ; * *, p<0.01 ;{ }^{*}, p<0.05$. 
models ${ }^{18}$, although the effect is much more pronounced in our case. Compared to $\mathrm{V} 1$, some discontinuities along the fibers are observed in V5, V10, and V20 specimens, which is in agreement with the topographical features (Fig. 1). Interestingly, it is noteworthy that contrary to the width of elastic fibers which no longer significantly evolves after V10, mainly due to degradation, the elasticity of the fibers is continually increasing during ageing. This can presumably be linked to the non-enzymatic post-translational modifications elastic fibers suffer during their whole life such as glycation ${ }^{9,24,25}$. Along with the change in the YM values for the elastic fibers, a significant increase in elasticity of the spaces between the fibers is also surprisingly observed, that could correspond to modifications in collagen and SMCs, with YM value of 178.6 $\pm 21.3 \mathrm{kPa}$ for V5, 316.3 $\pm 19 \mathrm{kPa}$ for $\mathrm{V} 10$, and $385.3 \pm 17.6 \mathrm{kPa}$ for V20. The SEM evolution clearly shows that the inter-fiber space phase is becoming more homogeneous with the ageing. Importantly, the increase of the YM moduli of the elastic fibers is in agreement with the aPWV experiments showing an increase of the velocity between the V1 and the V20 specimens (Table 1). Very interestingly, linear correlation study between aPWV and fiber relative Young's modulii showed a very strong correlation reinforcing our demonstration that the fibers elasticity increased during aging is a crucial determinant of the observed arterial stiffness. As stated before, aPWV, as an indirect method, do not allow to precisely quantify the local changes in arterial stiffness, contrary to AFM measurements which, thanks to the mapping of the elastic properties, allow to have a clear view of the behavior of both the elastic fibers and the elastic inter-fibers space. Our data clearly show better resolution and precision in the obtained results, compared to previous studies. This brings valuable information about elastic fibers evolution during ageing, but also that elastic inter-fiber space stiffens. If increasing during the whole life, EIF space presents the most striking modification during the first half of the life of the animals, as the elastic fibers width do. These innovative data could be obtained through the setting up of a highly efficient AFM technology. Moreover, our work showing a crucial involvement of ECM components stiffness, especially elastin, in the appearance of vascular stiffness during aging, presents valuable and complimentary data to a study demonstrating a role for endothelial cell stiffness increase during diabetes leading to endothelial dysfunction, a process highly increased during hyperglycemia that could participate to vascular stiffness ${ }^{26}$. Historically, non-oscillating AFM modes have been more utilized since they are based on a linear approach and allow for direct extraction of quantitative information. Up to very recently, with the development of high-speed force spectroscopy, they have suffered from a very long acquisition time and have been quite limited in terms of lateral resolution. With PFQNM mode, the AFM probe hammers the sample, which in return is deformed with a plastic and an elastic contribution; the force indentation data is mainly dominated by the permanent sample elasticity with negligible adhesion forces and very low viscosity. Such minimization is permitted by small approach velocities ${ }^{27}$ (generally below $1 \mu \mathrm{m} / \mathrm{s}$ ), which means in quasi static loading conditions. On the contrary, using high velocity approaches would cause the apparent $\mathrm{YM}$ to increase, due to much higher viscous effects ${ }^{28}$.
Murine models have been studied using such approaches, for instance for the studies of atherosclerotic plaques. Indeed, force mapping experiments conducted on $\mathrm{ApoE}^{-/}$mice have shown that the average YM on fibrotic areas was in the order of $10 \mathrm{kPa}$ whereas six up to twenty-five times higher on hypocellular fibrous caps. Lipidrich areas exhibited even higher variations ${ }^{15}$. On the other hand, there has been an increasing number of publications where AFM is used in non-contact mode. In that case, there is no real contact i.e. no indentation by the probe ${ }^{29}$ which can make the mechanical characterization easier ${ }^{30}$ and allow to minimize the loading force down to the piconewton (whereas it is more around the nanonewton in standard force spectroscopy) with an acquisition speed compared to those of tapping mode ${ }^{31}$ with cantilever oscillation occurring at a very high speed, from a few tens of Hertz up to the $\mathrm{GHz}$, depending on the technique. The first frequency-dependent measurements have been conducted on polymer gels ${ }^{32}$ and live cells ${ }^{33}$. For instance, Scanning Acoustic Microscopy (SAM) measures the propagation speed of longitudinal waves through a sample, which can be related to its YM. The cantilever is usually oscillated at a low amplitude. Since the acoustic wave speed depends on both the elastic modulus and the local density of the object, this technique can only characterize the sample elasticity indirectly. Nevertheless, it can be operated on any commercial AFM and thus has been often used to characterize local variations in the elastic properties of aorta ${ }^{12-14}$. Frequencymodulated AFM (FM-AFM), which also doesn't require any specific hardware, is also widely used on this type of sample; it detects small changes in the cantilever's resonance frequency, in response to the tip-sample interaction ${ }^{16}$. Such technique was recently used to investigate the mechanical properties of the medial layer (elastic lamellae and adjacent inter-lamellar space) of the ascending aorta from sheep of various ages (ranging from 18 months up to 8 years) and showed that the average $\mathrm{YM}$ was in the order of $43 \mathrm{kPa}$ for young specimens and increased up to $114 \mathrm{kPa}$ for older ones ${ }^{18}$. One must notice that the measured values are highly dependent on the animal model; for instance, rat ${ }^{34}$, sheep ${ }^{18}$ or human ${ }^{35}$, due to the difference in the vascular wall composition and organization. Also, the caliber of the arteries can strongly impact the elastic modulus (for instance ${ }^{36}$ vs. ${ }^{37}$ ). Each of those techniques has its pros and cons (for a more exhaustive comparison, $\mathrm{see}^{38}$ ) but the vast majority of these studies are based on the use of colloidal probes, ranging from $1^{37}$ to $12 \mu \mathrm{m}$ 15,18 . As a consequence, the returned $\mathrm{YM}$ only reflects the overall mechanical properties of the spot of interest (in that case, both FMAFM and AFM have a linear lateral resolution of 1 to $2 \mu \mathrm{m}$ ). In our case, PFT imageing offers the advantage of being used with standard AFM probes instead of latex beads. The resulting lateral resolution mainly depends on the tip geometry and allows for a direct comparison between local changes in topography and in mechanical properties, which means that on a single image, one can clearly distinguish from the elastic properties of an elastin fiber from those of the ECM. In terms of acquisition speed, it is comparable to tapping mode; and the loading force can be minimized down to a few tens of piconewtons (for instance when working on live cells, with the use of very low spring constant- cantilevers). Nevertheless, in the case of stiffer samples like aorta sections, it must be increased in order to 
sufficiently indent into the sample and get a valid mechanical response. With respect to the drive frequency, it can easily be changed from $250 \mathrm{~Hz}$ up to $4 \mathrm{~Hz}$. We chose to work as low as possible to minimize the viscous drag and increase as much as possible the tip-sample interaction time. Finally, the oscillation amplitude can also be manually changed. Since this type of sample is quite rough, the highest possible amplitude $(1 \mu \mathrm{m})$ was used.

\section{Experimental}

\section{Sample Preparation}

The aorta sections ( $n=9$ per mice) were obtained from 1, 5, 10 and 20 month-old C57/bl6 mice. Mouse experiments were performed in agreement with the European legislation on care and use of laboratory animals and were approved by the ethical committee of Reims Champagne-Ardenne. C57BI/6 mice ( $n=9$ for each conditions) were purchased from Janvier Labs (Le Genest-Saint-Isle, France). As shown in Fig. 1a, the samples were prepared as followed: after laparotomy, the organs were withdrawn so that the aorta could be dissected, then aorta rings without grease were cryofixed using Optimum Cutting Temperature (OCT, Tissue-Tek ${ }^{\circledR}$ O.C.T. Compound, Sakura ${ }^{\circledR}$

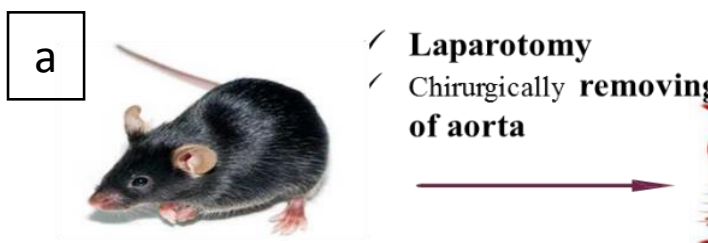

C57Bl/6 mice

(1 to 20 months old )

Cryostat cutting:

$16 \mu \mathrm{m}$ slices
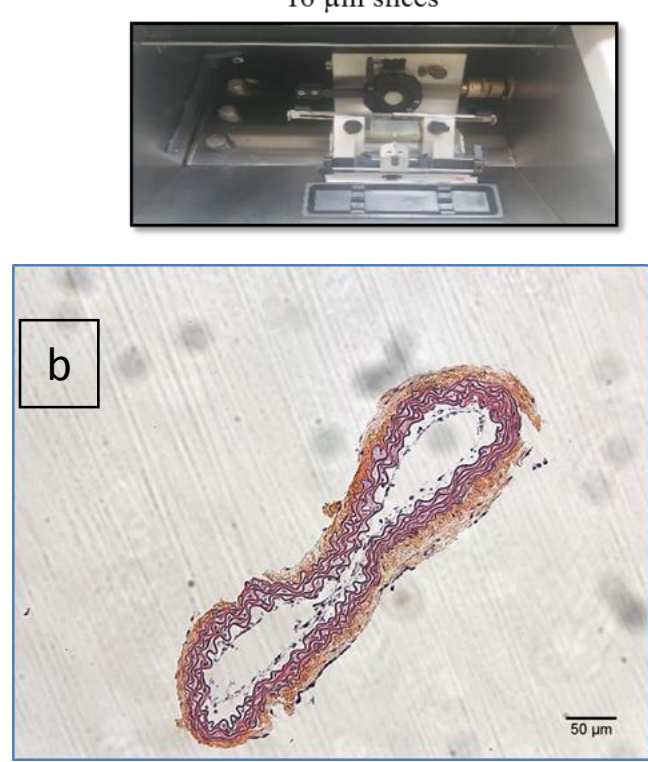

Finetek, VWR). The samples were kept at $-80^{\circ} \mathrm{C}$ prior to use. The cuts were made using a cryostat (Leica, Nanterre), the average thickness was ranging from 12 to $16 \mu \mathrm{m}$, and topped by $200 \mu \mathrm{l}$ of Krebs ( $\mathrm{NaCl} 118.5 \mathrm{mmol} / \mathrm{L}, \mathrm{KCl} 4.7 \mathrm{mmol} / \mathrm{L}, \mathrm{CaCl}_{2} 2.5 \mathrm{mmol} / \mathrm{L}$, $\mathrm{NaHCO}_{3} 25 \mathrm{mmol} / \mathrm{L}, \mathrm{MgSO}_{4} 1.2 \mathrm{mmol} / \mathrm{L}, \mathrm{KH}_{2} \mathrm{PO}_{4} 1.2 \mathrm{mmol} / \mathrm{L}$, and glucose $11 \mathrm{mmol} / \mathrm{L}, \mathrm{pH}$ 7.3-7.4) buffer for AFM observation at ambient temperature. In parallel to AFM measurements which have been performed on non-stained sections, hematoxylin/eosin (HES) staining was performed on sections from a same set (V1 to V20) allowing a better histological description of the samples. In this case, aortas were fixed immediately after removal in paraformaldehyde $4 \%$ and then paraffin embedded for cut and histological staining. Sections of $4 \mu \mathrm{m}$ were stained with hematoxylin-eosin and observed under an Olympus light microscope. As shown in Fig. 3a, the HES staining exhibit the lamellae surrounding the lumen of the vessel walls and the organization of the elastin sheets.

\section{Fluorescence Microscopy}

To analyze aortic elastin breaks, immediately after the sacrifice of mice, thoracic aortas were dissected and then directly frozen in liquid nitrogen. $4 \mu \mathrm{m}$ slices of OCT-embedded samples were rinsed in PBS for $5 \mathrm{~min}$ and conserved with FluorSave TM
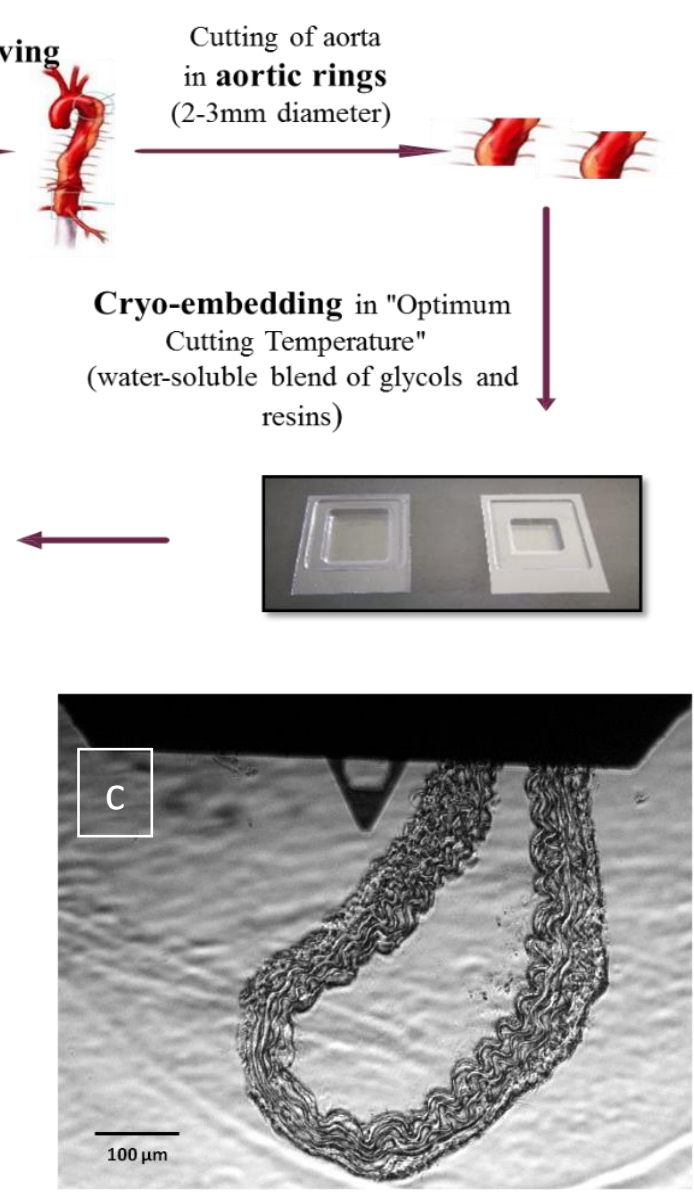

Figure 3. Summary of the protocol used for sample preparation and aortas analysis (a) schematic procedure for the aorta tissue preparation from the mice to the tissue cross sections. (b) HES staining of the aorta cross sections exhibiting the lamelae surrounding the lumen of the vessel walls and the regular organization of elastin sheets. (c) Optical image of a typical section studied by AFM. The AFM tip can be directly placed on the area of interest to perform high resolution topographical and nanomechanical imaging. 
Reagent (Calbiochem). Autofluorescence of elastin (excitation/emission: 410/500 nm) was evaluated using a Zeiss microscope (Axio A1).

\section{Pulse Wave Velocity}

Measurement of aortic pulse wave velocity (aPWV ; 10 mice per condition) was performed using the Doppler Flow Velocity system (Indus Instruments, Houston, Texas) ${ }^{39}$. Doppler signals were measured at the level of thoracic and abdominal aortas by using focused $10 \mathrm{MHz}$ and $20 \mathrm{MHz}$ Doppler probes, respectively. Signals were recorded and distance between the two probes measured, allowing aPWV calculation using the following formula: $\operatorname{aPWV}(\mathrm{m} / \mathrm{s})=$ distance $(\mathrm{m}) / \Delta \mathrm{t}(\mathrm{s})$ in which distance $=$ distance between the two probes and $\Delta t=$ averaged time between two ejection times.

\section{AFM Imaging}

A Bioscope Catalyst ${ }^{\mathrm{TM}}$ (Bruker, Billerica, USA) AFM coupled to a Nikon Eclipse Ti inverted microscope (Nikon, Tokyo, Japan) was used in this study. The glass slides with the tissues were put onto the AFM stage and observed with Bright Field illumination in order to locate the spots of interest, in particular the lamellae surrounding the lumen of the vessel walls (Fig. 3c). Then the AFM tip was approached towards the area to be analyzed. Given that the various samples exhibited a wide range of relative Young's moduli, and as proper probes have to be used in the PFQNM mode to get quantitative results, two types of AFM probes were used to image the elastin fibers: PFQNM-LC-A-CAL probes (Bruker, Billerica, USA) with pyramidal shape, having a nominal spring constant of $0.1 \mathrm{~N} / \mathrm{m}$ and a resonance frequency of $\sim 45 \mathrm{kHz}$ were used for the softest sample (V1) and DNP A probes (Bruker, Billerica, USA) with nominal spring constants of $0.35 \mathrm{~N} / \mathrm{m}$, and resonance frequencies of $65 \mathrm{kHz}$ were used for the stiffest samples (V5, V10, V20). These tips were chosen following the PFQNM protocol allowing the relative Young's Modulus to be measured. The two types of probes were chosen with characteristics as close as possible (geometry and tip radius) to limit the variations in the relative Young's modulus value that could come from differences in the tip parameters. The different probes have been carefully calibrated as previously described ${ }^{40}$. Prior to each measurement, the deflection sensitivity (by capturing three force curves on a noncompliant part of the sample and average the three corresponding slopes on the linear portions of the curves) and the spring constant (by tuning the cantilever at least $10 \mu \mathrm{m}$ away from the surface and spotting the resonance peak) were calculated for each probes. Regarding the tip radius, for each probe, they were determined before and after experiments using a reference titanium roughness sample (TipCheck Sample, Bruker, USA). The tip radius for the precalibrated PFQNM-LC-A-CAL was always around $65 \mathrm{~nm}$, consistent with the values provided by the provider, while for the DNP probes, the radius was measured between 20 and $60 \mathrm{~nm}$ depending on the probes. Variations in the tip radius and the cantilever spring constant that could occur during measurements were not significant after one sample scan and the probes were changed if some too important differences were observed before and after scans. The small changes in the tip radii that could occur were included into the standard deviation of the relative Young's Modulus. Images were captured in liquid with a resolution of 256 pixels per line. For PFQNM experiments, a PeakForce frequency of $0.25 \mathrm{kHz}$ was used in order to maximize the contact time between the tip and the sample with a PeakForce amplitude of $1 \mu \mathrm{m}$. The loading force was adjusted to few nanonewtons. A small approach velocity minimised the contribution of viscosity to the mechanical response. Thus, the hydrodynamic damping hysteresis was almost suppressed and had no impact of the Young's Modulus measurements. Indeed, a small approach velocity allowed to minimise the impact of the viscosity in the mechanical response of the tip 27,28 . The distance synchronization parameter was manually and constantly adjusted over time so that the turnaway point of each force curve was exactly at the $(x, y)$ maximum position. Regarding the relative Young's modulus calculation and the topographical measurements, for each mice age, three mice were studied and at least 3 different tissues coming from the same mouse were prepared. At the end, between 15 and 20 images per conditions were analyzed. For all the aorta sections, their thickness were superior to $10 \mu \mathrm{m}$ (in the order of 14 to $16 \mu \mathrm{m}$ ). As the indentation depth was always limited to around $100 \mathrm{~nm}$, it could be considered that the substrate is not contributing to the measured relative YM. The topographical images were treated using Nanoscope Analysis $v 1.8$ to extract the width of elastin fibers. At least 150 fiber sections were analyzed for each ageing conditions to obtain an average value of the fiber width. For the determination of the relative YM, as the retraction and the approach curves were similar whatever the scanned area (see Fig. SI), showing a negligible contribution of the viscosity, the retraction force curves were extracted from chosen area from the PFQNM images. Since arterial cross section thicknesses $(10 \mu \mathrm{m})$ were largely superior to the indentation depth (around $100 \mathrm{~nm})$, the tissues could be considered as a semi-infinite substrate and then the classical Hertz model (1) was used to fit the force curves:

$F(\delta)=\frac{4}{3} \frac{E}{1-v^{2}} \cdot \sqrt{R} \cdot \delta^{3 / 2}$

where $E$ is the relative Young's modulus, $v$ is the Poisson ratio of the sample ( 0.5 for our samples considered as incompressible), $R$ is the nominal radius of the tip. As it is known that the relative Young's modulus variations could be due to topography effects, great care was taken to confirm the absence of correlation between the topography and the nanomechanical map (see Fig. SI). Particularly, when analyzing the mechanical properties and extracting forcedistance, areas of low rugosity (inferior to $15 \mathrm{~nm}$ ) were chosen so be sure that the variations in relative Young's Modulus were not linked to the surface roughness. Taking into account the assumptions to extract nanomechanical data ${ }^{19,41}$, the average relative Young's modulus $E$ was then computed for each curve by fitting the forceversus-indentation curve to the model.

\section{Statistical Analysis}

Results were expressed as mean \pm SEM. Statistical significance was evaluated using the Student's $t$ test. $p$ values of less than 0.05 were considered statistically significant. 


\section{Conclusions}

In this study, topographical and nanomechanical mapping using the PeakForce QNM mode were used to image aorta sections from 1, 5, 10 and 20 months-old C57/bl6- mice. Thanks to high resolution and the possible quantification of the modifications in the fiber structure and elastic properties, the changes in the mechanical and topographical information could be related to the ageing process. A direct connection between local changes in structure and mechanical properties of both elastin fibers and the intermediate matrix, and the specimen ages were successfully evidenced. Unlike other well-established microscopy techniques (fluorescence, histology) the PFQNM mode, after carefully preparing the samples, allows extracting mechanical data at the same resolution as on the height channel, since a force curve is captured and recorded for each image pixel, which allowed us to capture mechanical images at an unprecedented resolution. The potentiality of this technique applied to the local characterization of elastic fibers should lead to major breakthrough in the studies of cardiovascular-related diseases at nanoscale. The precise quantification of the fiber elasticity could allow to better follow fiber remodeling and to predict the plaque rupture in many atherosclerotic diseases.

\section{Conflicts of interest}

There are no conflicts to declare.

\section{Acknowledgements}

The authors thank the FEDER, Région Grand Est, and DRRT Grand Est for their support of the Nano'Mat platform. We are also thankful to C. Feuillie (CBMN UMR CNRS 5248) for the fruitful discussions.

\section{Funding}

This works have been supported by internal grants from the Université de Reims Champagne-Ardenne, the region Grand Est, and the CNRS.

\section{Ethical Statement}

Mice experiments were performed in agreement with the European legislation on care and use of laboratory animals. All animal procedures were moreover performed in accordance with the Guidelines for Care and Use of Laboratory Animals of Reims Champagne-Ardenne University and approved by the Animal Ethics Committee of Reims Champagne-Ardenne.

\section{Author contributions}

A.Ber., A. W., A.H. L.D., and M.M. participated to the scientific discussions, analyzed the data and wrote the manuscript. A.Ber. carried out the AFM experiments. A.W., A.H., L.G. and C.P. were responsible for animal maintenance and prepared the histological samples. S.B., B.R.C. and P.M. performed aPWV measurement. A. Ben. and H.S. performed fluorescence studies.
A. Ber. and M.M. designed the AFM experiment and L.D. the biological part. S.J., P.G., F.T. and L.M. edited and reviewed the manuscript. L.D. and M.M. designed and coordinated the project. All authors read and approved the final manuscript.

1. A. Wahart, T. Hocine, C. Albrecht, A. Henry, T. Sarazin, L. Martiny, H. El Btaouri, P. Maurice, A. Bennasroune, B. Romier-Crouzet, S. Blaise and L. Duca, The FEBS journal, 2019, 286, 2980-2993.

2. R. Nakanishi, L. Baskaran, H. Gransar, M. J. Budoff, S. Achenbach, M. Al-Mallah, F. Cademartiri, T. Q. Callister, H. J. Chang, K. Chinnaiyan, B. J. W. Chow, A. DeLago, M. Hadamitzky, J. Hausleiter, R. Cury, G. Feuchtner, Y. J. Kim, J. Leipsic, P. A. Kaufmann, E. Maffei, G. Raff, L. J. Shaw, T. C. Villines, A. Dunning, H. Marques, G. Pontone, D. Andreini, R. Rubinshtein, J. Bax, E. Jones, N. Hindoyan, M. Gomez, F. Y. Lin, J. K. Min and D. S. Berman, Hypertension, 2017, 70, 293-299.

3. J. E. Wagenseil and R. P. Mecham, Journal of cardiovascular translational research, 2012, 5, 264-273.

4. H. Sage, Comparative biochemistry and physiology. $B$, Comparative biochemistry, 1983, 74, 373-380.

5. B. S. Brooke, A. Bayes-Genis and D. Y. Li, Trends in cardiovascular medicine, 2003, 13, 176-181.

6. C. M. Kielty, M. J. Sherratt and C. A. Shuttleworth, Journal of cell science, 2002, 115, 2817-2828.

7. S. D. Shapiro, S. K. Endicott, M. A. Province, J. A. Pierce and E. J. Campbell, The Journal of clinical investigation, 1991, 87, 1828-1834.

8. L. Duca, S. Blaise, B. Romier, M. Laffargue, S. Gayral, H. EI Btaouri, C. Kawecki, A. Guillot, L. Martiny, L. Debelle and P. Maurice, Cardiovascular research, 2016, 110, 298-308.

9. E. Konova, S. Baydanoff, M. Atanasova and A. Velkova, Experimental gerontology, 2004, 39, 249-254.

10. M. F. O'Rourke, Vascular medicine, 2007, 12, 329-341.

11. M. J. Sherratt, Age, 2009, 31, 305-325.

12. R. Akhtar, Artery research, 2014, 8, 1.

13. H. K. Graham, R. Akhtar, C. Kridiotis, B. Derby, T. Kundu, A. W. Trafford and M. J. Sherratt, Mechanisms of ageing and development, 2011, 132, 459-467.

14. N. Lopez-Andres, A. Rousseau, R. Akhtar, L. Calvier, C. Inigo, C. Labat, X. Zhao, K. Cruickshank, J. Diez, F. Zannad, P. Lacolley and P. Rossignol, Hypertension, 2012, 60, 563-573.

15. P. Tracqui, A. Broisat, J. Toczek, N. Mesnier, J. Ohayon and L. Riou, Journal of structural biology, 2011, 174, 115-123.

16. M. J. Higgins, J. E. Sader and S. P. Jarvis, Biophysical journal, 2006, 90, 640-647.

17. J. Huynh, N. Nishimura, K. Rana, J. M. Peloquin, J. P. Califano, C. R. Montague, M. R. King, C. B. Schaffer and C. A. Reinhart-King, Science translational medicine, 2011, 3, 112 ra122.

18. R. Akhtar, H. K. Graham, B. Derby, M. J. Sherratt, A. W. Trafford, R. S. Chadwick and N. Gavara, Journal of the mechanical behavior of biomedical materials, 2016, 64, 1017.

19. R. Garcia, Chemical Society reviews, 2020, DOI: 10.1039/d0cs00318b.

20. R. Newton, M. Delguste, M. Koehler, A. C. Dumitru, P. R. Laskowski, D. J. Muller and D. Alsteens, Nature protocols, 2017, 12, 2275-2292.

21. H. Schillers, I. Medalsy, S. Hu, A. L. Slade and J. E. Shaw, Journal of molecular recognition : JMR, 2016, 29, 95-101. 
22. S. E. Greenwald, The Journal of pathology, 2007, 211, 157172.

23. P. A. Janmey and C. A. McCulloch, Annual review of biomedical engineering, 2007, 9, 1-34.

24. Y. Yamamoto, N. Sakata, J. Meng, M. Sakamoto, A. Noma, I. Maeda, K. Okamoto and S. Takebayashi, Nephrology, dialysis, transplantation : official publication of the European Dialysis and Transplant Association - European Renal Association, 2002, 17, 630-636.

25. K. Zarkovic, P. Larroque-Cardoso, M. Pucelle, R. Salvayre, G. Waeg, A. Negre-Salvayre and N. Zarkovic, Redox biology, 2015, 4, 109-117.

26. M. Targosz-Korecka, M. Jaglarz, K. E. Malek-Zietek, A. Gregorius, A. Zakrzewska, B. Sitek, Z. Rajfur, S. Chlopicki and M. Szymonski, Scientific reports, 2017, 7, 15951.

27. I. Kang, D. Panneerselvam, V. P. Panoskaltsis, S. J. Eppell, R. E. Marchant and C. M. Doerschuk, Biophysical journal, 2008, 94, 3273-3285.

28. G. Francius, J. Hemmerle, J. Ohayon, P. Schaaf, J. C. Voegel, C. Picart and B. Senger, Microscopy research and technique, 2006, 69, 84-92.

29. N. Gavara and R. S. Chadwick, Nature methods, 2010, 7, 650-654.

30. D. C. Lin, D. I. Shreiber, E. K. Dimitriadis and F. Horkay, Biomechanics and modeling in mechanobiology, 2009, 8, 345-358.

31. X. Zhao, R. Akhtar, N. Nijenhuis, S. J. Wilkinson, L. Murphy, C. Ballestrem, M. J. Sherratt, R. E. Watson and B. Derby, IEEE transactions on ultrasonics, ferroelectrics, and frequency control, 2012, 59, 610-620.

32. R. E. Mahaffy, C. K. Shih, F. C. MacKintosh and J. Kas, Physical review letters, 2000, 85, 880-883.

33. R. E. Mahaffy, S. Park, E. Gerde, J. Kas and C. K. Shih, Biophysical journal, 2004, 86, 1777-1793.

34. M. F. Berry, A. J. Engler, Y. J. Woo, T. J. Pirolli, L. T. Bish, V. Jayasankar, K. J. Morine, T. J. Gardner, D. E. Discher and H. L. Sweeney, American journal of physiology. Heart and circulatory physiology, 2006, 290, H2196-2203.

35. S. R. Barrett, M. P. Sutcliffe, S. Howarth, Z. Y. Li and J. H. Gillard, Journal of biomechanics, 2009, 42, 1650-1655.

36. T. Matsumoto, T. Goto, T. Furukawa and M. Sato, Journal of biomechanics, 2004, 37, 807-815.

37. T. Oie, Y. Murayama, T. Fukuda, C. Nagai, S. Omata, K. Kanda, H. Yaku and Y. Nakayama, Journal of artificial organs : the official journal of the Japanese Society for Artificial Organs, 2009, 12, 40-46.

38. R. Akhtar, M. J. Sherratt, J. K. Cruickshank and B. Derby, Materials today, 2011, 14, 96-105.

39. A. K. Reddy, S. Madala, A. D. Jones, W. A. Caro, J. F. Eberth, T. T. Pham, G. E. Taffet and C. J. Hartley, Ultrasound in medicine \& biology, 2009, 35, 2042-2054.

40. C. Heu, A. Berquand, C. Elie-Caille and L. Nicod, Journal of structural biology, 2012, 178, 1-7.

41. Y. F. Dufrene, T. Ando, R. Garcia, D. Alsteens, D. MartinezMartin, A. Engel, C. Gerber and D. J. Muller, Nature nanotechnology, 2017, 12, 295-307. 